www.nature.com/ja

\title{
Spread of $c f r$ gene among staphylococci conferring resistance to linezolid in a patient under treatment
}

\author{
Concepción Pérez-Jorge ${ }^{1}$, María-Carolina Isea-Peña ${ }^{1}$, Sarah Heilii, ${ }^{1,2}$ and Jaime Esteban ${ }^{1}$
}

The Journal of Antibiotics (2012) 65, 151-152; doi:10.1038/ja.2011.130; published online 21 December 2011

Keywords: acquisition; cfr; linezolid; resistance; Staphylococcus aureus; Staphylococcus epidermidis; treatment

Linezolid was the first member of the family of oxazolidinones to be introduced in clinical practice. This antibiotic has proven to be very effective against most of the Gram-positive organisms, including vancomycin-resistant or vancomycin-intermediate methicillin-resistant Staphylococcus aureus (MRSA) and vancomycin-resistant Enterococcus sp. ${ }^{1}$ Development of resistance was described only a few years after its introduction in clinical practice., ${ }^{2,3}$ Development of resistance in these reports was due to G2576T mutation in the 23S rRNA gene, and it appeared to be an uncommon finding. ${ }^{2-4}$ However, the first outbreak of a linezolid-resistant MRSA due to a different resistance mechanism has recently been described: the acquisition of $c f r$ gene, which also confers chloramphenicol, 16C macrolides, streptogramin A and clindamycin resistance. ${ }^{5}$ Previously the $c f r$ gene was described in other staphylococcal species of animal origin. ${ }^{6}$ Moreover, other cases of $c f r$-mediated linezolid resistance in S. aureus and S. epidermidis strains isolated from human infections have also been published. ${ }^{4,7}$ Here we report a case of acquisition of linezolid resistance from MRSA that was related to the development of chloramphenicol/clindamycin resistance with the parallel isolation of one linezolid-resistant S. epidermidis strain.

The patient was a 73-year-old woman who underwent an aortic valve replacement. Three weeks after surgery, the patient experienced fever, inflammatory symptoms in the surgical wound, thoracic instability, and cardiac and renal failure. The patient was then diagnosed with acute mediastinitis, and a linezolid-susceptible MRSA strain was isolated from two blood cultures (day 1). The patient was then treated with linezolid ( $600 \mathrm{mg}$ b.i.d. intravenous), and day 18 , medication was changed to oral therapy with clindamycin $(300 \mathrm{mg}$ b.i.d.) and linezolid (600 mg b.i.d.). Two weeks (day 32) after this modification, the disease persisted, and new cultures were performed (one from a wound exudate and four blood cultures). Linezolid-resistant MRSA strains were isolated from a wound exudate and from two blood cultures samples, and the linezolid-resistant strain of S. epidermidis was isolated from one blood culture mixed with one linezolid-resistant MRSA and in two additional blood cultures in pure culture.
Antimicrobial susceptibility was performed by means of the disk-diffusion method using the disk-plate assay according to the EUCAST protocols. ${ }^{8}$ MICs were subsequently performed by Etest (bioMérieux, Marcy l'Etoile, France) using strips of linezolid, vancomycin and daptomycin following also the EUCAST indications. ${ }^{8}$ Detection of the $c f r$ gene was performed by using previously described primers ${ }^{9}$ and protocols. ${ }^{5}$ A $c f r$-positive MRSA strain (kindly provided by Dr JJ Picazo ${ }^{5}$ ) was used as a positive control. Plasmid analysis was performed using an alkaline lysis previously described protocol. ${ }^{10}$

A randomly amplified polymorphic DNA assay was performed according to protocols described previously. ${ }^{11,12}$ We considered strains as belonging to the same clone if identical profiles were obtained with at least three different primer sets. We used three randomly selected strains of methicillin-resistant, linezolid-susceptible S. aureus from different sources (one blood culture and two wound exudates from different patients) were used as controls.

The entire study was performed according to the Ethical Committee requirements of our institution.

One linezolid-susceptible MRSA strain was isolated from blood cultures before linezolid treatment (day 1) and three additional linezolid-resistant MRSA were isolated after treatment from blood cultures (two strains) and wound exudates (1 strain) (day 32). Three linezolid-resistant methicillin-resistant S. epidermidis (MRSE) strains were isolated from blood cultures, one blood culture with MRSA and two additional blood cultures without.

Table 1 shows susceptibility to selected antibiotics. API STAPH profiles were identical in all MRSA strains (code no. 6736153). RAPD analysis showed that electrophoretic profiles of all MRSA isolates were identical with all the primer sets, but different from that which was obtained from the previously described $S$. aureus strains used as controls. The $c f r$ gene was detected in all the linezolid-resistant strains (both S. aureus and S. epidermidis), but not in the linezolid-susceptible MRSA strain isolated in the first positive blood cultures. No plasmids were detected in all the S. aureus and S. epidermidis strains. 
Table 1 Antimicrobial susceptibility of clinical isolates of Staphylococcus $s p$

\begin{tabular}{lcccccc}
\hline & \multicolumn{4}{c}{ Disk-plate (interpretation of inhibition zone) } & & E-Test (MIC mg /-1) \\
\cline { 2 - 6 } Strain & Oxacillin & Cefoxitin & Chloramphenicol & Clindamycin & Linezolid & Daptomycin \\
\hline $\mathrm{a}$ & $\mathrm{R}$ & $\mathrm{R}$ & $\mathrm{S}$ & $\mathrm{S}$ & 0.75 & 0.094 \\
$\mathrm{~b}$ & $\mathrm{R}$ & $\mathrm{R}$ & $\mathrm{R}$ & $\mathrm{R}$ & 16 & 0.75 \\
$\mathrm{C}$ & $\mathrm{R}$ & $\mathrm{R}$ & $\mathrm{R}$ & $\mathrm{R}$ & $>256$ & 0.125 \\
\hline
\end{tabular}

Abbreviations: R, resistant; S, susceptible.

(a) Linezolid-susceptible methicillin-resistant S. aureus; (b) linezolid-resistant methicillin-resistant S. aureus; (c) linezolid-resistant S. epidermidis.

Therapy of MRSA has been changed during the past years due to new findings in the development of resistance. Vancomycin has been the elective therapy for these patients for many years, but new data have recently emerged concerning this antibiotic, as patients infected with strains which have MIC $\geqslant 2 \mathrm{mgl}^{-1}$ were shown to have higher mortality than those with MIC $<1 \mathrm{mgl}^{-1}$ when they were treated with vancomycin. ${ }^{13}$ It is currently recommended that the use of vancomycin for MRSA strains with MIC between $1-2 \mathrm{mgl}^{-1}$ should be undertaken with caution, and alternative therapies should be considered. ${ }^{14}$

Although linezolid has been used extensively for the treatment of MRSA and other Gram-positive infections, development of resistance has been claimed to be a rare finding. ${ }^{4}$ The first cases reported were due to mutations in the $23 \mathrm{~S}$ rRNA gene, which lead to a change in the target of the antibiotic and were not transferable to other strains. ${ }^{3}$ However, the development of resistance due to the acquisition of the $c f r$ gene increases concern because the $c f r$ gene can be transferred to other strains and can spread in outbreaks, leading to important complications when providing therapy to infected patients. ${ }^{5}$

In this report, we have demonstrated that linezolid-susceptible and -resistant MRSA belongs to the same clone, which leads us to conclude that the resistance gene was acquired during treatment. The appearance of resistance against clindamycin, chloramphenicol and linezolid initially suggested the presence of a $c f r$ gene, and this finding was corroborated by PCR. The absence of plasmids suggests also the presence of a transposable element that would be inserted in the chromosome. In our case, linezolid-resistant MRSE strains carrying the $c f r$ gene were also isolated. We speculated about the possibility of genetic transfer of this gene between the strains of different species. It is possible that the linezolid-resistant MRSA strain acquired the $c f r$ gene from linezolid-resistant MRSE in the patient, with the resulting therapeutic failure during linezolid treatment, although other possible sources for this gene cannot be ruled out. Nevertheless, because S. epidermidis strains showed higher degree of linezolid resistance, it is possible that other mechanisms were involved in such resistance in these strains.

The importance of therapy duration for the acquisition of resistance is unknown in this case. Our patient was treated over 32 days, receiving a combination of linezolid and clindamycin, and selection of resistance against both the drugs appeared in the second set of cultures. Interestingly, the patient did not improve while undergoing therapy, so it seems probable that development of resistance occurred at the beginning of the therapy, and not after a long period of treatment.
In conclusion, we report the spread of the $c f r$ gene between MRSA and MRSE in a patient receiving linezolid treatment for MRSA infection. This fact reinforces the need for strict control of antibiotics in the hospital environment. RAPD can be used as a rapid method for molecular epidemiological characterization of $S$. aureus even with strains with different susceptibility patterns.

\section{ACKNOWLEDGEMENTS}

The work was funded by a grant from the MCYT (CONSOLIDER-INGENIO 2010 CSD 2008-0023 FUNCOAT).

1 Diekema, D. J. \& Jones, R. N. Oxazolidinone antibiotics. Lancet 358, 1975-1982 (2001).

2 Tsiodras, S. et al. Linezolid resistance in a clinical isolate of Staphylococcus aureus. Lancet 358, 207-208 (2001).

3 Gales, A. C., Sader, H. S., Andrade, S. S., Lutz, L., Machado, A. \& Barth, A. L. Emergence of linezolid-resistant Staphylococcus aureus during treatment of pulmonary infection in a patient with cystic fibrosis. Int. J. Antimicrob. Agents 27, 300-302 (2006).

4 Farrell, D. J., Mendes, R. E., Ross, J. E. \& Jones, R. N. Linezolid surveillance program results for 2008 (LEADER Program for 2008). Diagn. Microbiol. Infect. Dis. 65, 392-403 (2009).

5 Morales, G. et al. Resistance to linezolid is mediated by the cfr gene in the first report of an outbreak of linezolid-resistant Staphylococcus aureus. Clin. Infect. Dis. 50, 821-825 (2010).

6 Schwarz, S., Werckenthin, C. \& Kehrenberg, C. Identification of a plasmid-borne chloramphenicol-florfenicol resistance gene in Staphylococcus sciuri. Antimicrob. Agents Chemother. 44, 2530-2533 (2000).

7 Mendes, R. E., Deshpande, L. M., Castanheira, M., DiPersio, J., Saubolle, M. A. \& Jones, R. N. First report of $c f r$-mediated resistance to linezolid in human staphylococcal clinical isolates recovered in the United States. Antimicrob. Agents Chemother. 52, 2244-2246 (2008).

8 EUCAST. Breakpoints tables for interpretation of MICs and zone diameters (2011). URL: http://www.eucast.org/clinical breakpoints/. Access date: 10 September 2011.

9 Kehrenberg, C. \& Schwarz, S. Distribution of florfenicol resistance genes fexA and cfr among chloramphenicol-resistant Staphylococcus isolates. Antimicrob. Agents Chemother. 50, 1156-1163 (2006).

10 Sambrook, J. \& Russell, D. Molecular Cloning: a Laboratory Manual 3rd edn Vol 1, (CSHL Press, New York, 2011).

11 Kurlenda, J., Grinholc, M., Jasek, K. \& Wegrzyn, G. RAPD typing of methicillin-resistant Staphylococcus aureus: a 7-year experience in a Polish hospital. Med. Sci. Monit. 13, MT13-MT18 (2007)

12 Reinoso, E., Bettera, S., Frigerio, C., DiRenzo, M., Calzolari, A. \& Bogni, C. RAPD-PCR analysis of Staphylococcus aureus strains isolated from bovine and human hosts. Microbiol. Res. 159, 245-255 (2004).

13 Takesue, Y. et al. Clinical characteristics of vancomycin minimum inhibitory concentration of $2 \mu \mathrm{g} / \mathrm{ml}$ methicillin-resistant Staphylococcus aureus strains isolated from patients with bacteremia. J. Infect. Chemother. 17, 52-57 (2011).

14 Haque, N. Z. et al. Relationship of vancomycin minimum inhibitory concentration to mortality in patients with methicillin-resistant Staphylococcus aureus hospitalacquired, ventilator-associated, or health-care-associated pneumonia. Chest 138 , 1356-1362 (2010) 